
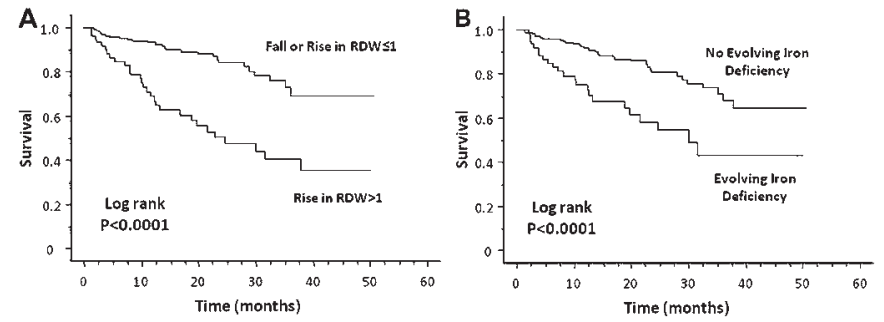

Abstract 107 Figure 1

Conclusions An expanding RDW and evolving iron deficiency over time predict an amplified risk of death in CHF and could be utilised for risk stratification or therapeutically targeted to improve outcomes.

\section{4D-FLOW CMR DEMONSTRATES THE REGIONAL DISTRIBUTION OF AORTIC FLOW DISTURBANCE IN MARFAN SYNDROME}

doi:10.1136/heartjnl-2011-300198.108

${ }^{1} \mathrm{~A}$ Pitcher, ${ }^{1} \mathrm{~T}$ E Cassar, ${ }^{1} \mathrm{~J}$ Suttie, ${ }^{1} \mathrm{~J} \mathrm{M}$ Francis, ${ }^{2} \mathrm{P}$ Leeson, ${ }^{3} \mathrm{E}$ Blair, ${ }^{4} \mathrm{~B}$ P Wordsworth, ${ }^{5} \mathrm{~J} C$ Forfar, ${ }^{6} \mathrm{M}$ Markl, ${ }^{1} \mathrm{~S} N \mathrm{~N}$ Neubauer, ${ }^{7} \mathrm{~S}$ E Petersen. ${ }^{1}$ Oxford Centre for Clinical Magnetic Resonance Imaging, Oxford, UK; ${ }^{2}$ Department of Cardiovascular Medicine, University of Oxford, Oxford, UK; ${ }^{3}$ Department of Clinical Genetics, Churchill Hospital, Oxford, UK; ${ }^{4}$ Nuffield Department of Medicine, University of Oxford, Oxford, UK; ${ }^{5}$ John Radcliffe Hospital, Oxford, UK; ${ }^{6}$ University Hospital, Freiburg, Germany; ${ }^{7}$ Centre for Advanced Cardiovascular Imaging, William Harvey Research Institute, London, UK

Background Marfan syndrome (MFS) commonly leads to progressive aortic dilation, aneurysm formation and aortic dissection, particularly at the aortic sinuses ( $\sim 60 \%$ of dissections), and descending thoracic aorta ( $\sim 30 \%$ of dissections). Abnormal aortic blood flow patterns may contribute to the enlargement and dissection of an inherently weak aorta, or to late complications after aortic dissection.

Methods 18 patients with MFS ( 3 with a prior history of aortic dissection and aortic root surgery, 15 with no such history) and 18 healthy volunteers matched for age, sex and height underwent CMR at 3T, using a time-resolved 3-dimensional flow technique. The aorta was segmented into regions on the basis of anatomic features (Abstract 108 figure 1A). Each segment was visualised using streamlines (Abstract 108 figure 1B) and particle traces, and was rated as normal or abnormal, (defined as the presence of turbulent flow vortices) and, where abnormal, extent of abnormality was classified on a 4-point scale determined by the extent of radial involvement of the aortic lumen. Wall shear stress (WSS) quantification was undertaken at predefined aortic locations (Abstract 108 figure $1 \mathrm{~A})$

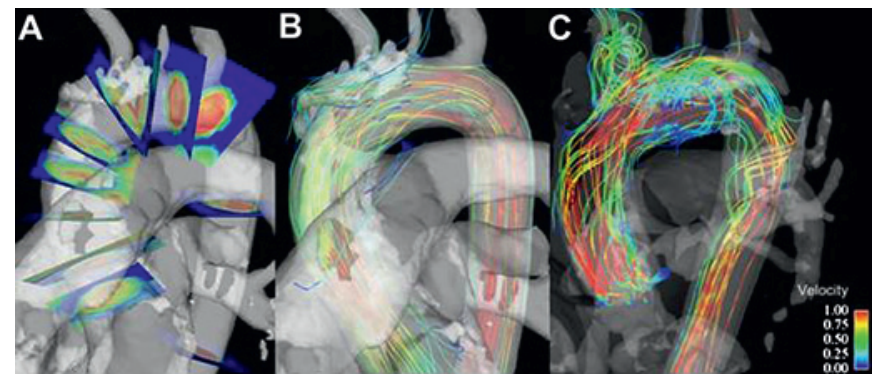

Abstract 108 Figure 1 A. Planes for aortic segmentation and WSS quantification. B. Flow visualisation in a healthy volunteer. C. Flow visualisation in a patient with prior aortic dissection fulfilling the Ghent Criteria for Marfan syndrome.
Results Significant vortical flow in any segment (defined as flow disturbance occupying more than one half of the aortic lumen) was present in all patients with MFS, but in only $7 / 18$ controls $(p<0.0005)$. The severity of flow disturbance was greater in MFS patients than controls (median severity score 3 for Marfan patients, 1 for controls, $\mathrm{p}<0.0005$ ). There was marked regional variation in the prevalence of major flow disturbance (Abstract 108 figure 2), with the sinuses of Valsalva and proximal descending aorta being most frequently affected. Prior repaired aortic dissection was associated with marked abnormalities of blood flow (Abstract 108 figure 1C), with corresponding increases in axial WSS within the true lumen of the dissected aorta (typical axial WSS in the dissected ascending aorta was $+0.9 \mathrm{~N} / \mathrm{m}^{2}$, compared to $+0.54 \mathrm{~N} / \mathrm{m}^{2}$ in healthy controls). Aortic flow disturbance in MFS was of one of three types: Type A: flow disturbance confined to the sinuses of Valsalva, Type B: flow disturbance confined to the proximal descending aorta, Type C: flow disturbance in both the sinuses of Valsalva and the proximal descending aorta.

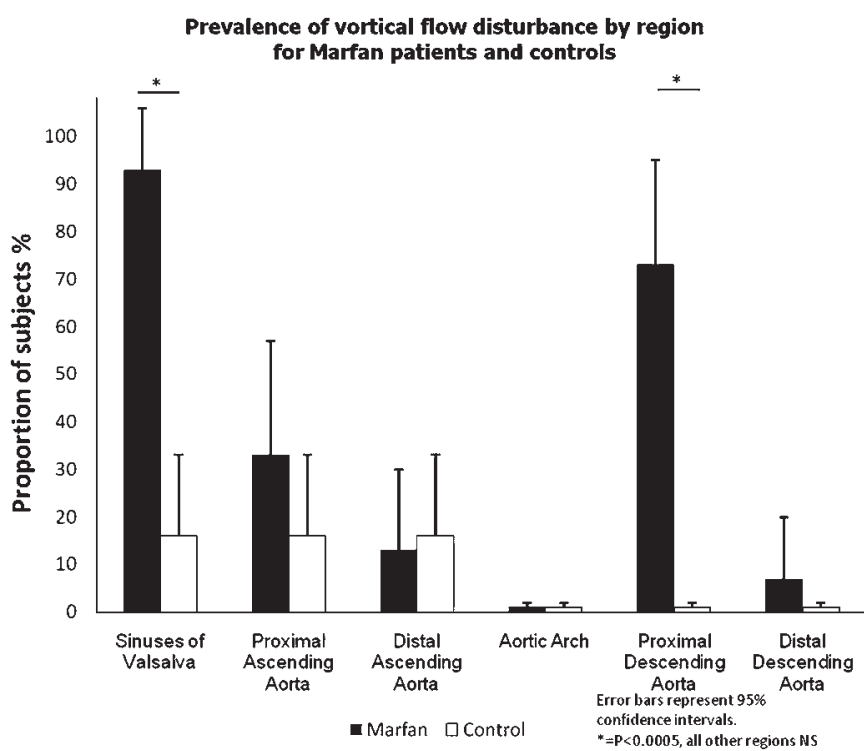

Abstract 108 Figure 2 Prevalence of vortical flow disturbance occupying $>50 \%$ luminal diameter for each aortic region for Marfan patients and controls.

Conclusion Patients with MFS commonly show aortic flow disturbance. The sinuses of Valsalva and proximal descending aorta are most frequently affected. Flow disturbance can be categorised into one of three categories, and we anticipate that flow abnormalities within a segment will predict progressive aortic dilation and dissection in an ongoing follow-up study.

\section{3T MRI OF ACUTE ATHEROSCLEROTIC PLAQUE RUPTURE AND DOWNSTREAM EMBOLIC INJURY}

doi:10.1136/heartjnl-2011-300198.109

A C Lindsay, L Biasiolli, J M Lee, I Kylintireas, H Watt, W Kuker, A Handa M D Robson, S Neubauer, J Kennedy, R P Choudhury. University of Oxford, Oxford, UK

Introduction Luminal stenosis is a poor predictor of the risk posed by any given atherosclerotic plaque, therefore current angiographic imaging techniques cannot reliably determine which patients are most likely to suffer future ischaemic events. However, MRI may be able to detect features of atherosclerotic plaque rupture that have been associated with an increased risk of recurrent atherothrombosis. 